\title{
IASSIST 2011 - CALL FOR PAPERS
}

The 37th International Association for Social Science Information Services and Technology (IASSIST) annual conference will be hosted by Simon Fraser University and University of British Columbia and will be held in Vancouver, Canada, May 31 - June 3, 2011.

The theme of this year's conference is Data Science Professionals: a Global Community of Sharing. Social science benefits from professional practices that enable sharing of data, information, and knowledge with a global community. This theme is intended to stimulate discussions about ways in which sharing data, information, and knowledge can contribute to research and to professional practices that enable scientific progress. Submissions are encouraged that offer improvements for creating, documenting, submitting, describing, disseminating, and preserving scientific research data.

We seek submissions on the theme outlined above, and encourage conference participants to propose papers and sessions that would be of interest to themselves and other attendees. Below is a sample of possible topics that may be considered: * open data and the development of knowledge communities

- Data sharing, access and management in the future

- Identifying and reducing barriers to data sharing- Issues of confidentiality in sharing

- Sharing professional data science skills, knowledge, \& techniques within \&across discipline

- Citation of research data and persistent identifiers

- Metadata facilitating data sharing

- Emerging research infrastructures and data sharing

- New data partnerships in knowledge communities

- Sharing resources and data through social networks

- Identifying user needs and customizing data services to meet the needs

- The evolving data librarian profession

- Data science practices that support global use and understanding of research data

- Open (linked) data and digital repositories

- Preservation for sharing, recovering data for contemporary use

Proposals on other topics related to the conference theme will be considered too. Papers will be selected from a wide range of subjects to ensure a broad balance of topics.

The Program Committee welcomes proposals for:

- Individual presentations (typically 15-20 minutes)

- Sessions, which could take a variety of formats (e.g. a set of three or four presentations, a discussion panel, a discussion with the audience, etc.)

- Posters/demonstrations for the poster session

- Workshops (pre-conference workshops that blend lecture and hands-on instruction). [Note: A separate call for workshops is forthcoming.] 


\section{|ASSIST 2011}

Again this year we would be interested in receiving submissions for presentations in formats successfully introduced in last year's conference, in particula

- Pecha Kucha (a presentation of 20 slides shown for 20 seconds each, with a heavy emphasis on visual content).

- Round table discussions (as these are likely to have limited spaces, an explanation of how the discussion will be shared with the wider group should form part of the proposal).

Session formats are not limited to these ideas and session organizers are welcome to suggest other formats. Proposals for complete sessions should list the organizer or moderator and possible participants; the session organizer will be responsible for securing both session participants and a chair.

All submissions should include the proposed title and an abstract no longer than 200 words. Longer abstracts will be returned to be shortened before being considered. Please note that all presenters are required to register and pay the registration fee for the conference; registration for individual days will be available.

A web form for submission of proposals will be available on the conference web site on October 18, 2010.

Deadline for submission: November 29, 2010.

Notification of acceptance: February 3, 2011.

Please note that the Conference Program Committee may not be able to accept all proposals.

Conference papers will be considered for articles in the IASSIST Quarterly. This applies to individual papers as well as selections of papers from sessions that could form special issues of the IASSIST Quarterly.

For more information about the conference, including travel and accommodation, see the conference web site at:

http://www.rdl.sfu.ca/IASSIST/

Online conference registration is scheduled to open in early February, 2011.

Make plans to come to Vancouver for IASSIST 2011 : 31 May - 3 June 2011!

Questions may be sent to the Program Planning Co-Chairs,

Bob Downs, Ernie Boyko and Tuomas J. Alateräi at iassist2011@gmail.com 